\title{
Electrostatic Precipitation in the Martian Environment
}

\author{
Hiroyuki Kawamoto ${ }^{1}$, Shunpei Kojima ${ }^{2}$ and Shuta Inari ${ }^{3}$ \\ Department of Applied Mechanics and Aerospace Engineering, Waseda University \\ 4-4-1, Okubo, Shinjuku, Tokyo 169-8555, Japan
}

\begin{abstract}
Because the Martian atmosphere is mainly composed of $\mathrm{CO}_{2}$, electrochemical reduction of oxygen from $\mathrm{CO}_{2}$ is one of the most efficient methods of extracting the oxygen that is indispensable for astronauts to breathe and can be used as an oxidant for rocket engines. To realize a reliable oxygen production system, a large amount of $\mathrm{CO}_{2}$ gas must be accumulated and compressed in an electrolytic conversion system. However, because dust is presented in the Martian atmosphere because of dust storms, there is a concern that it could damage the oxygen conversion system or cause it to malfunction. Thus, the presence of a dust removal system is necessary before $\mathrm{CO}_{2}$ gas can be introduced into the system. To this end, we are developing an electrostatic precipitator suitable for use in the low-pressure Martian atmosphere. We first constructed a coaxial cylindrical precipitator consisting of a wire and cylinder electrodes. In our preliminary investigation, the efficiency was at most $60 \%$ without corona discharge, and almost no dust was collected at the corona discharge region in the lowpressure (700 Pa) atmosphere. A low pressure of 700 Pa was chosen to simulate the Martian atmosphere. In this case, dust was collected on the surface of the wire electrode, contrary to the case for a pressure of $10^{5} \mathrm{~Pa}(1 \mathrm{~atm})$. The mechanism of these phenomena was investigated by the direct observation and numerical calculation of particle motion in the precipitator. It was clarified that the low charge density of particles in the low-pressure atmosphere caused a relatively large dielectrophoresis force compared to the Coulomb force, and the particles were attracted to the wire electrode. The poor performance in the low-pressure atmosphere was caused by the low charge density and low electrostatic field owing to the low limited voltage. Although the performance was degraded very rapidly in low pressure atmosphere due to the deposition of dust on the fine wire electrode, it was very easy to vibrate the wire electrode and to remove dust on the wire.
\end{abstract}

\section{Nomenclature}

$C_{c} \quad=$ Cunningham correction factor (-)

$d \quad=$ diameter of particle $(\mathrm{m})$

$E_{x} \quad=$ electrostatic field in the radial direction $(\mathrm{V} / \mathrm{m})$

$g=$ gravitational acceleration $\left(9.8 \mathrm{~m} / \mathrm{s}^{2}\right)$

$I_{c} \quad=$ corona current $(\mathrm{A})$

$m \quad=$ mass of particle $(\mathrm{kg})$

$P \quad=$ pressure (Torr)

$q \quad=$ charge of particle $(\mathrm{C})$

$r \quad=$ radial coordinate from the center of the wire electrode $(\mathrm{m})$

$r_{i} \quad=$ radius of wire electrode $(\mathrm{m})$

$r_{o} \quad=$ inner radius of cylinder electrode $(\mathrm{m})$

$T \quad=$ temperature $\left({ }^{\circ} \mathrm{C}\right)$

$V \quad=$ applied voltage $(\mathrm{V})$

\footnotetext{
${ }^{1}$ Professor, Dept. of Applied Mechanics and Aerospace Engineering, Waseda University, 4-4-1, Okubo, Shinjuku, Tokyo 169-8555, Japan/kawa@waseda.jp.

${ }^{2}$ Student, Dept. of Applied Mechanics and Aerospace Engineering, Waseda University, 4-4-1, Okubo, Shinjuku, Tokyo 169-8555, shun-waseda@fuji.waseda.jp.

${ }^{3}$ Student, Dept. of Applied Mechanics and Aerospace Engineering, Waseda University, 4-4-1, Okubo, Shinjuku, Tokyo 169-8555, Japan/Inashu91_0820@ruri.waseda.jp.
} 


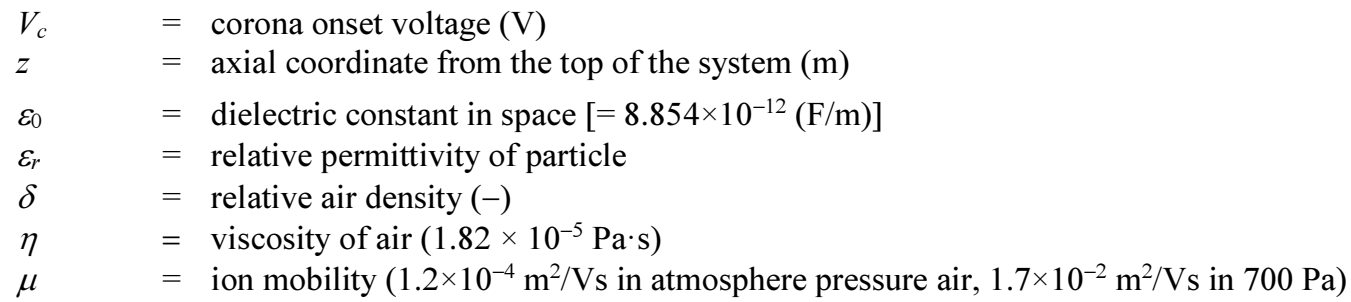

\section{Introduction}

$\mathrm{T}^{\circ}$ realize long-term manned exploration of Mars, in-situ resource utilization (ISRU) is necessary, both to manage the risk and reduce the cost of transporting resources from Earth. ISRU requires a number of processes, such as the drilling, collection, classification, and chemical processing of the Martian regolith, to extract and synthesize oxygen, water, and any metals. ${ }^{1-7}$ Because the Martian atmosphere is primary composed of carbon dioxide, electrochemical reduction of oxygen from carbon dioxide is believed to be one of the most efficient methods to extract the oxygen that is a requirement for astronauts to breathe, and can be used as an oxidant for rocket engines. To realize a reliable oxygen production system, a large amount of $\mathrm{CO}_{2}$ gas must be accumulated and compressed in an electrolytic conversion system. However, because dust particles in the range of 2-10 $\mu \mathrm{m}$ in diameter are present in the Martian atmosphere because of dust storms and dust devils,, 8 there are concerns that the dust could damage the oxygen conversion system or cause it to malfunction. Thus, the implementation of a dust removal system is necessary before $\mathrm{CO}_{2}$ gas can be introduced in the system. To this end, we are developing an electrostatic precipitator that is suitable for the use in the low-pressure Martian atmosphere. ${ }^{10-13}$

The electrostatic precipitator consists of a wire electrode and a grounded cylinder electrode surrounding the wire. ${ }^{11}$ Gas including the airborne dust is injected into the cylinder electrode. When a high voltage is applied to the wire, a corona discharge takes place near the wire and generates ions that traverse between the wire and cylinder electrodes. These particles are then charged with the same polarity as the wire electrode. The charged particles move toward the outer electrode owing to the electrostatic field between the electrodes, and the charged dust particles are deposited there. The captured particles are removed from the collecting cylinder electrode mechanically. This technology is mainly utilized in industrial applications such as the diesel engines and thermal power generation plants, to capture fine particles in the exhaust gas, and because the technology has several advantages such as low pressure drop, low power consumption, and high collection efficiency, it is suitable for the use in space applications.

We investigated the preliminary performance of the electrostatic precipitator in the low-pressure atmosphere to verify its utility for the reliable and efficient oxygen production system required for ISRU in the Martian environment.

\section{Experimental}

\section{A. Experimental Setup}

Figure 1 (a) shows a schematic drawing of the experimental setup. A coaxial wire-to-cylinder system was installed in a vacuum chamber. A high positive DC voltage was applied to the wire by using a high-voltage power supply (Matsusada Precision, HEOPS-10BS), and the cylinder electrode was grounded. The diameter of the tungsten wire electrode was $0.1 \mathrm{~mm}$, and two aluminum cylinders with inner diameters of $30 \mathrm{~mm}$ and $50 \mathrm{~mm}$ were used for the parametric experiment. In addition to the wire-to-cylinder system, a wire-to-plate system, as shown in Figure 1 (b), was prepared to observe particle motion directly from the lateral side of the wire. The distance between the parallel plates was $50 \mathrm{~mm}$ and the width of the plates was $80 \mathrm{~mm}$. The length of the cylinders and plates was $150 \mathrm{~mm}$. Dust particles were fed to the upper part of the cylinder using a vibration feeder, i.e., a container was laterally vibrated using an unbalanced motor, and dust in the container was fed through a $124-\mu \mathrm{m}$ opening mesh attached to the bottom of the container. The feed rate was controlled by adjusting the applied voltage to the motor. The vacuum chamber was evacuated using a rotary pump, and pressure in the chamber was measured by a pirani gauge (ULVAC, GLD-136). The dust collection rate was determined by the weight of the dust that passed though the cylinder with the application of voltage divided by that without applying voltage. For each experiment, the weight of the dust was measured after an operational duration of one minute using an electric balance (Mezler, WMC25 -25) that is appropriate for use in a vacuum environment. Because the performance was degraded very rapidly in low pressure atmosphere due to the deposition of dust on the fine wire electrode, the dust collection rate was measured at the very beginning of the operation. Details on this feature and countermeasure are described in the section V. 


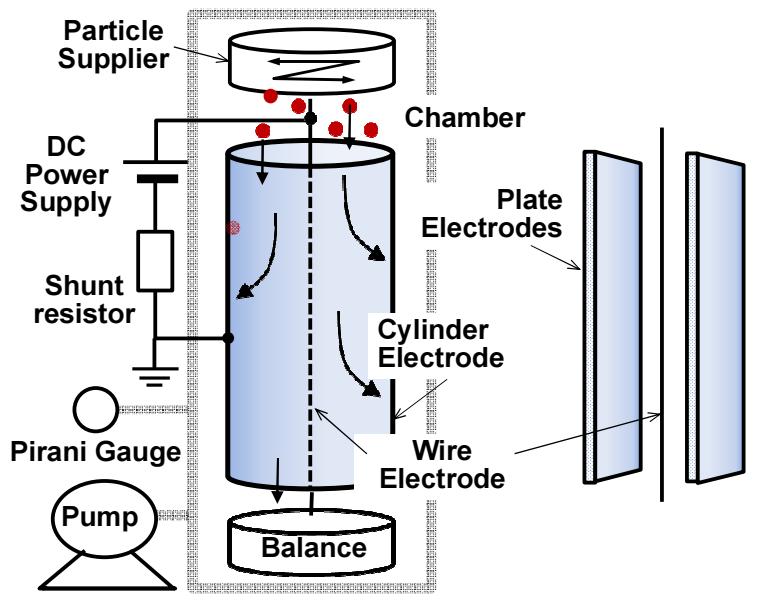

(a) (b)

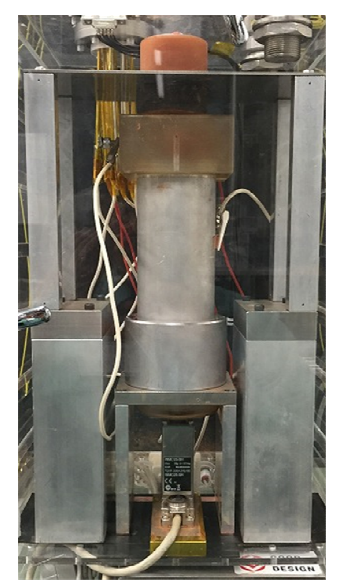

(c)

Figure 1. Experimental setup. (a) Schematic drawing of electrostatic precipitator (Wire-to-cylinder electrode system is installed in the vacuum chamber), (b) wire-to-plate electrode system, (c) Photograph of setup (Wireto-cylinder electrode system is installed in the chamber)

Table 1. Properties of test powder, actual Martian regolith, and Martian regolith simulant.

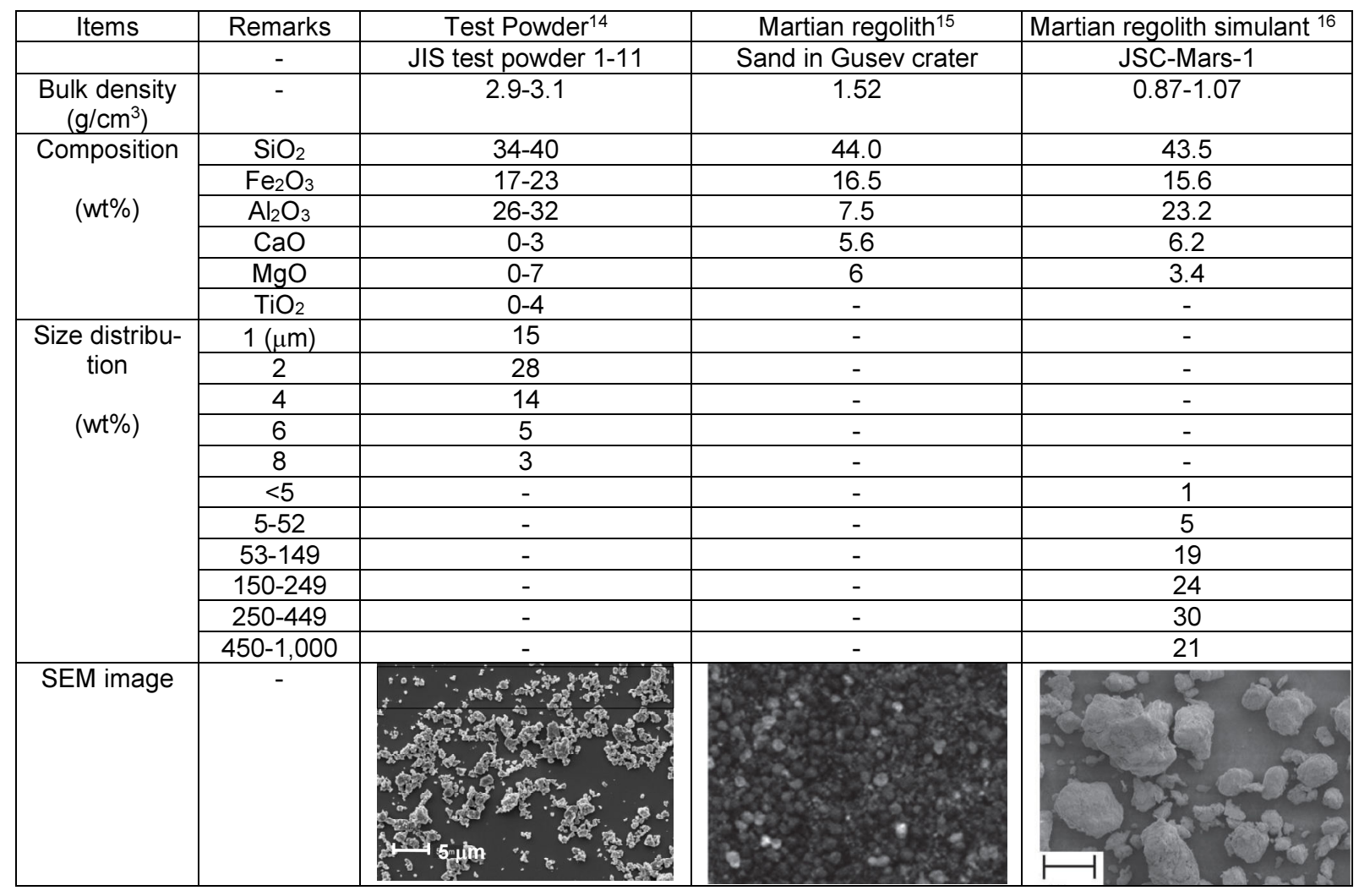

\section{B. Dust Used for Experiment}

Because it is assumed that actual airborne dust is much smaller than that on the surface of Mars, we used small test powder, the characteristics of which are listed in Table 1. It is reported that Martian particle size of dust suspended for long period of time is $1-2 \mu \mathrm{m}$ and that raised into the atmosphere by wind and dust devil is lower than 5 $\mu \mathrm{m} .{ }^{11}$ It is not exact same but similar to the test sample. The chemical composition itself does not affect the motion of particles but physical and electrostatic properties of dust, such as the charge, permittivity, specific gravity, and 
size of particle, determine the motion of particles in the electrostatic precipitator, nevertheless the composition of the test powder was also similar to the Martian regolith.

\section{Fundamental Characteristics}

Before conducting the dust removal experiment, the voltage-current characteristics of the wire-to-cylinder system were measured as shown in Figure 2. The current was measured by dividing the voltage drop in a shunt resistor inserted between the power supply and the wire electrode by the resistance. The parameters in consideration were; the inner diameter of the outer cylindrical electrode and pressure of the air. At voltages lower than the threshold (4.7-5.1 kV for normal atmospheric pressure and $0.68-0.75 \mathrm{kV}$ at $700 \mathrm{~Pa}$ ), no substantial current flowed in the air gap. However, at voltages higher than the threshold voltage, a discharge current of the order of several microamperes was measured, and a weak luminescence was observed at the wire electrode at the same time. That happened because of the occurrence of corona discharge. The associated current and luminescence were stable, and the discharge was silent. When the applied voltage was increased, the magnitude of corona current increased, and the discharge mode shifted to a spark discharge. These characteristics are typical in a wire-to-cylinder system. ${ }^{17}$

The corona onset voltage $V_{c}(\mathrm{~V})$ is calculated by the following equation.

$$
V_{c}=7200 \delta\left(1+\frac{0.0301}{\sqrt{r_{i} \delta}}\right) r_{i} \log _{10} \frac{r_{o}}{r_{i}}, \quad \delta=\frac{P}{760} \frac{298}{T+273} \text { (relative air density) }
$$

where $r_{i}$ is the radius of the wire electrode $(\mathrm{m}), r_{o}$ is the inner radius of the cylinder electrode $(\mathrm{m}), P$ is the pressure (Torr), and $T$ is the temperature $\left({ }^{\circ} \mathrm{C}\right)$. The calculated corona onset voltage at $10^{5} \mathrm{~Pa}(1 \mathrm{~atm}, 760$ Torr) was $4.68 \mathrm{kV}$ for the case of the $30-\mathrm{mm}$ inner diameter cylinder and $5.10 \mathrm{kV}$ for the case of the $50-\mathrm{mm}$ inner diameter cylinder. The onset voltage at $700 \mathrm{~Pa}$ was $0.68 \mathrm{kV}$ for the case of the $30-\mathrm{mm}$ inner diameter cylinder and $0.75 \mathrm{kV}$ for the case of the 50-mm inner diameter cylinder. These values were consistent with the measured values shown in Figure 2. The corona onset voltage in a low-pressure atmosphere was lower compared to that a pressure of $10^{5} \mathrm{~Pa}$.

The corona current $I_{c}(\mathrm{~A})$ is calculated by the following equation.

$$
I_{c}=\frac{4 \pi \varepsilon_{0} \mu}{r_{o} \ln \left(r_{o} / r_{i}\right)} V\left(V-V_{c}\right)
$$

where $\varepsilon_{0}$ is the dielectric constant in space $\left(8.854 \times 10^{-12} \mathrm{~F} / \mathrm{m}\right), \mu$ is the ion mobility $\left(1.2 \times 10^{-4} \mathrm{~m}^{2} / \mathrm{Vs}\right.$ at an air pressure of $10^{5} \mathrm{~Pa}, 1.7 \times 10^{-2} \mathrm{~m}^{2} / \mathrm{Vs}$ at $\left.700 \mathrm{~Pa}\right)$, and $V$ is the applied voltage $(\mathrm{V})$. As shown in Figure 2 , the calculated values coincided fairly well to the measured values.

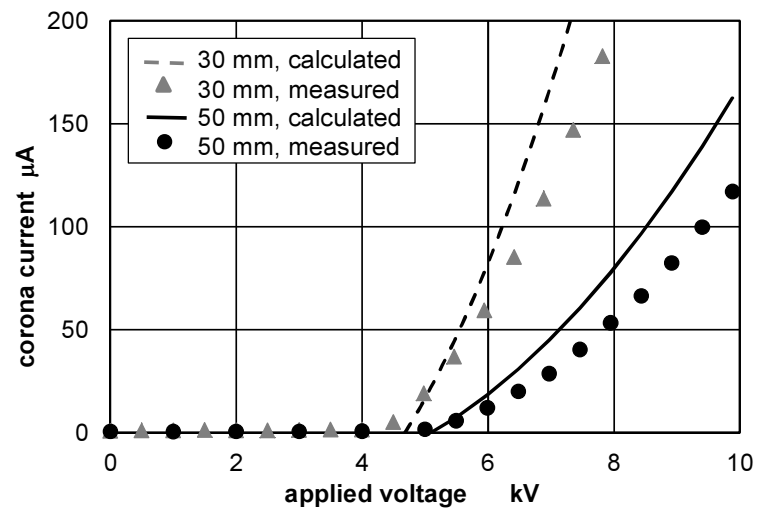

(a) in atmosphere pressure $\left(10^{5} \mathrm{~Pa}\right)$ air

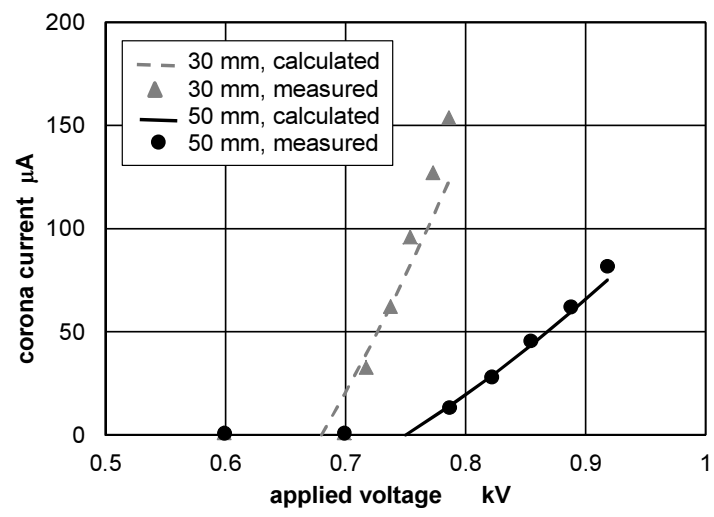

(b) in 700 Pa air

Figure 2. Measured and calculated $V-I$ curves in the wire-to-cylinder system (parameter: inner diameter of cylinder electrode.

Figure 3 shows the measured dust collection rate in the wire-to-cylinder system. The rate increased with an increase of the applied voltage, and was around $70-80 \%$ at the atmosphere pressure when the applied voltage was just below the corona onset voltage, as shown in Figure 3 (a). The rate was a little high when the inner diameter of the cylinder electrode was small. A very high rate, approximately $100 \%$, was realized by the application of a high volt- 
age to the wire electrode that generated the corona discharge. Dust was collected on the inner surface of the cylinder electrode.

On the other hand, at a lower pressure (700 Pa), which was selected to simulate the Martian atmosphere, the efficiency was at most 50-60\% without corona discharge and almost no dust was collected in the corona discharge region, as shown in Figure 3 (b). The applied voltage at $700 \mathrm{~Pa}$ was very low compared to that at earth's atmospheric pressure, owing to the low corona onset voltage. Dust was collected on the surface of the wire electrode contrary to the case for $10^{5} \mathrm{~Pa}$.

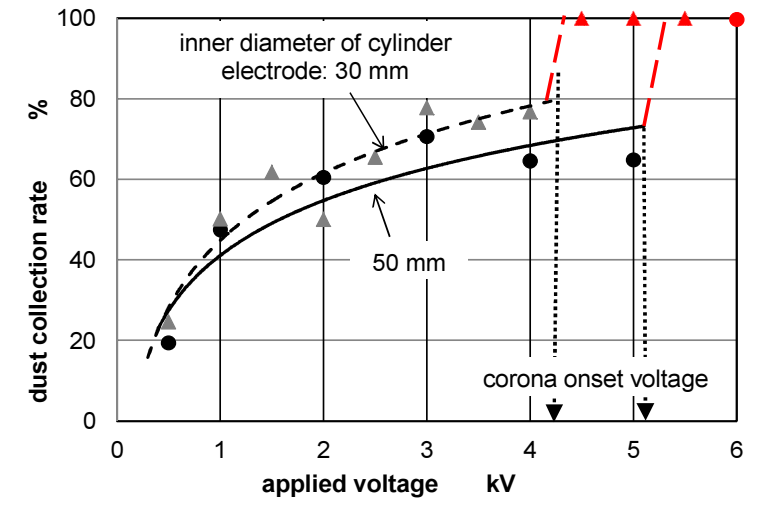

(a) in atmosphere pressure $\left(10^{5} \mathrm{~Pa}\right)$ air

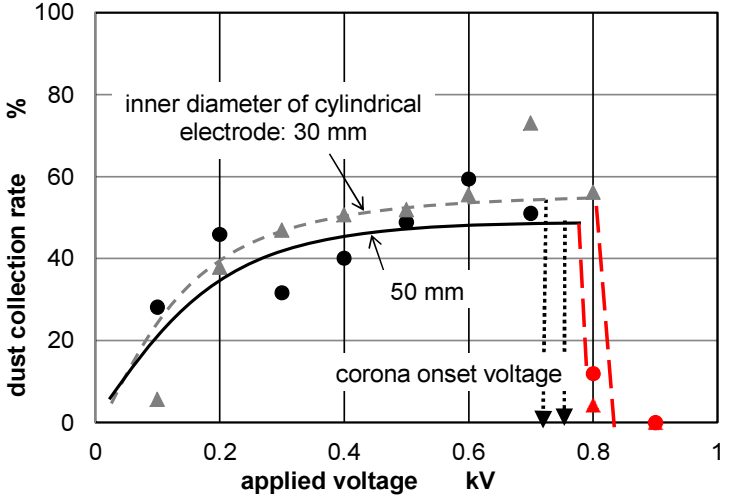

(b) in 700 Pa air

Figure 3. Measured dust collection rate in the wire-to-cylinder system.

The wire-to-parallel plate electrode system was prepared to investigate the different characteristics of the particle motion between the dust collection at atmosphere pressure and that in the low-pressure atmosphere. This is because the motion of particles could be observed directly from the lateral side of this system using a high-speed video camera. Figure 4 shows the measured V-I curves in the wire-to-plate system at $10^{5} \mathrm{~Pa}$ and at $700 \mathrm{~Pa}$. The characteristics were common to those for the wire-to-cylinder system shown in Figure 2.

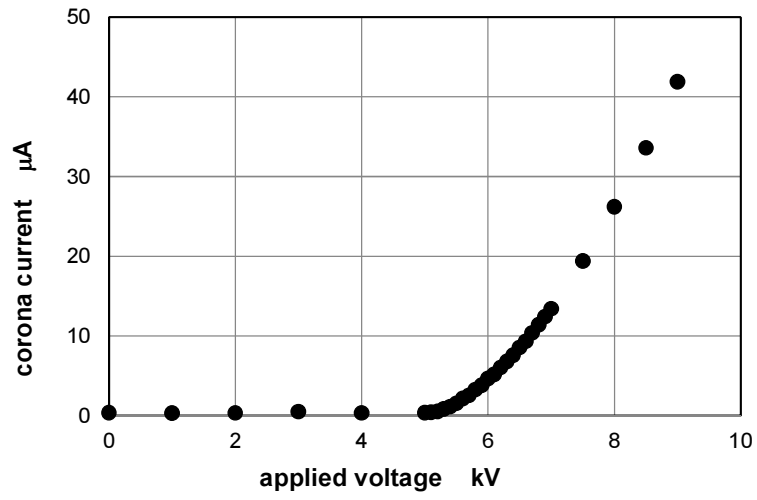

(a) in atmosphere pressure $\left(10^{5} \mathrm{~Pa}\right)$ air

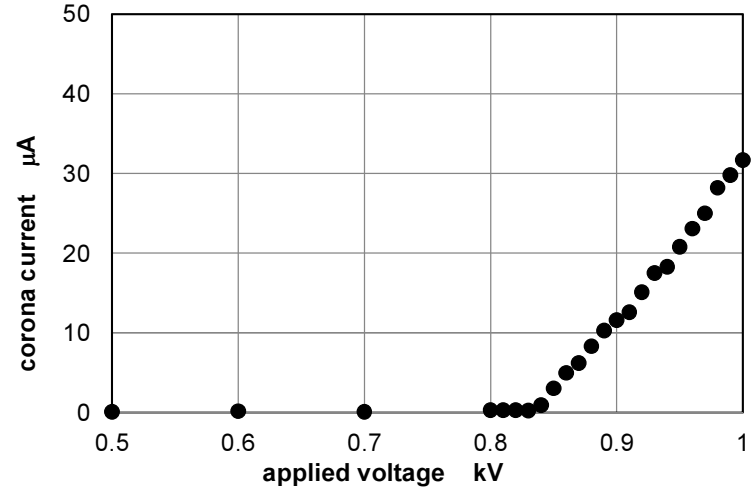

(b) in 700 Pa air

Figure 4. Measured V-I curves in the parallel wire-to-plate system.

Figure 5 shows the measured dust collection rate in the wire-to-plate system. The fundamental characteristics are common to those in the wire-to-cylinder system shown in Figure 3. The rate increased with an increase in the applied voltage, and was around $80 \%$ at $10^{5} \mathrm{~Pa}$ at the applied voltage just below the corona onset voltage. A very high rate, of approximately $90-100 \%$, was realized by applying a higher voltage to the wire electrode that generated the corona discharge. Dust was collected on the inner surface of the plate electrodes. However, in the low-pressure (700 $\mathrm{Pa}$ ) atmosphere, although the efficiency was $80 \%$ without a corona discharge, almost no dust was collected in corona discharge region. Dust was collected on the surface of the wire electrode unlike the case for $10^{5} \mathrm{~Pa}$. 


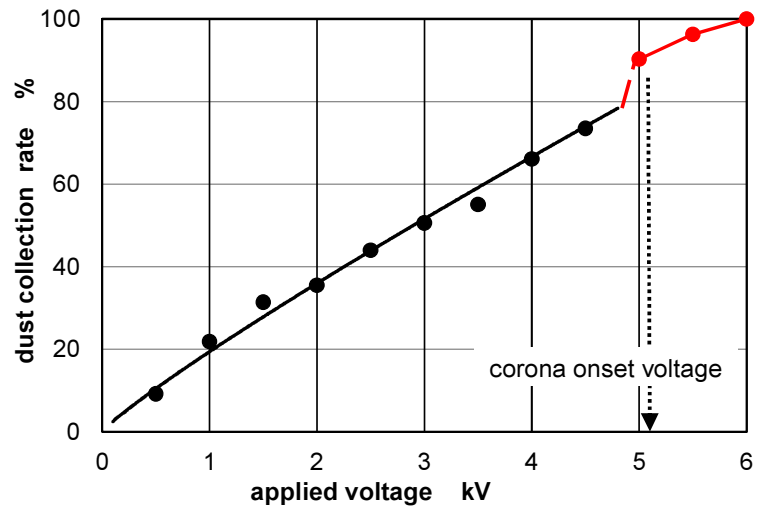

(a) in atmosphere pressure $\left(10^{5} \mathrm{~Pa}\right)$ air

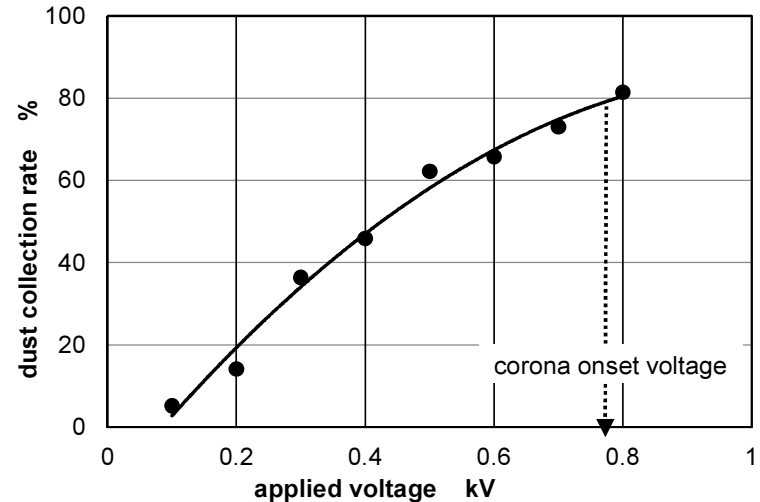

(b) in $700 \mathrm{~Pa}$ air

Figure 5. Measured dust collection rate in the parallel wire-to-plate system.

\section{Particle Trajectories}

\section{A. Measured Trajectories and Motion of Particles}

Figure 6 summarizes particle motions observed from the lateral side of the wire electrode in the wire-to-plate system. Typical particle trajectories are illustrated in the figure.

(1) At atmospheric pressure $\left(10^{5} \mathrm{~Pa}\right)$, applied voltage lower than corona onset $(0.6-4.5 \mathrm{kV})$

Particles introduced near the wire electrode moved to the wire electrode owing to the Coulomb force and dielectrophoresis force applied to particles, and then touched the wire. The particle was charged positively at contact, and repelled from the positive wire electrode to the negative plate electrode. The repelling speed of charged particle was high when the applied voltage was high, owing to the high electrostatic field, and the particles were attracted to the plate electrode. On the other hand, particles introduced near the plate electrodes moved to the plate electrodes owing to the Coulomb force (if the particle was charged positively), and then attracted to the plate. In both cases, particles were deposited on the surface of the plate electrodes as observed. Because some particles were charged negatively, the dust collection rate was not $100 \%$.

Figure 7 shows the accelerations of randomly selected particles in the system. The accelerations were deduced from the video images by using the motion analyzing software TEMA (Image Systems). It was confirmed that particles near the wire electrode were attracted to the wire electrode. Figure 7 (a) shows that particles located at approximately $8 \mathrm{~mm}$ from the wire were attracted to the wire electrode when the applied voltage was lower than corona onset at $10^{5} \mathrm{~Pa}$.

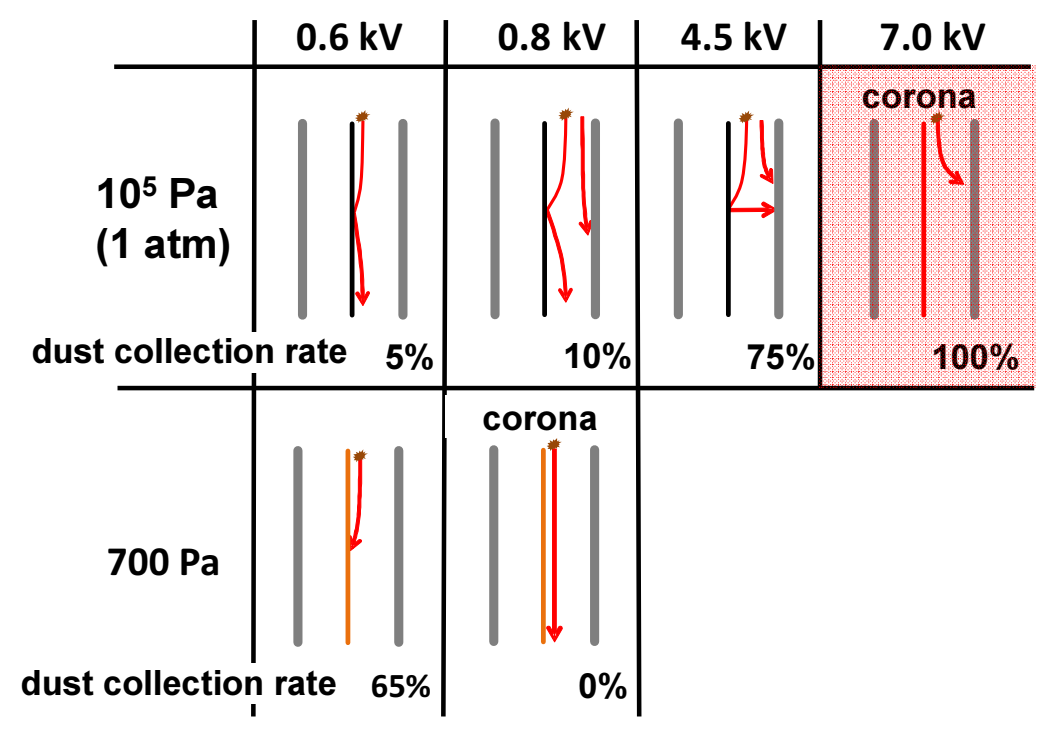

Figure 6. Summary of particle trajectories in the wire-to-plate system.

6

International Conference on Environmental Systems 
(2) At atmospheric pressure $\left(10^{5} \mathrm{~Pa}\right)$, applied voltage higher than corona onset $(7 \mathrm{kV})$

Almost all particles were attracted to the plate electrode, because both positive and negative particles were charged positively when they came in contact with the positive ions generated by the corona discharge. This was consistent to the experimental results wherein almost $100 \%$ particles were deposited on the surface of the grounded electrode at the corona discharge region at $10^{5} \mathrm{~Pa}$.

(3) At low pressure (700 Pa), applied voltage lower than corona onset $(0.6 \mathrm{kV})$

Particles introduced near the wire electrode moved to the wire electrode owing to the Coulomb force and dielectrophoresis force applied to particles, and then touched the wire. The characteristics of particles' motion were similar to those at $10^{5} \mathrm{~Pa}$ so far. However, the contacted particles were not repelled but rather deposited on the wire electrode. This caused the low dust collection rate.

Figure 7 (b), the trajectories of particles at $700 \mathrm{~Pa}$ analyzed by TEMA, showed that particles located at approximately $2 \mathrm{~mm}$ from the wire electrode were attracted to the wire electrode at $700 \mathrm{~Pa}$. The influential area at $700 \mathrm{~Pa}$ was much smaller than that at $10^{5} \mathrm{~Pa}$. The mechanism of these features is discussed in the subsequent section.

(4) At low pressure (700 Pa), applied voltage higher than corona onset $(0.8 \mathrm{kV})$

Almost all particles dropped without contact with the wire or plate electrodes, and therefore, the dust collection rate was almost $0 \%$.

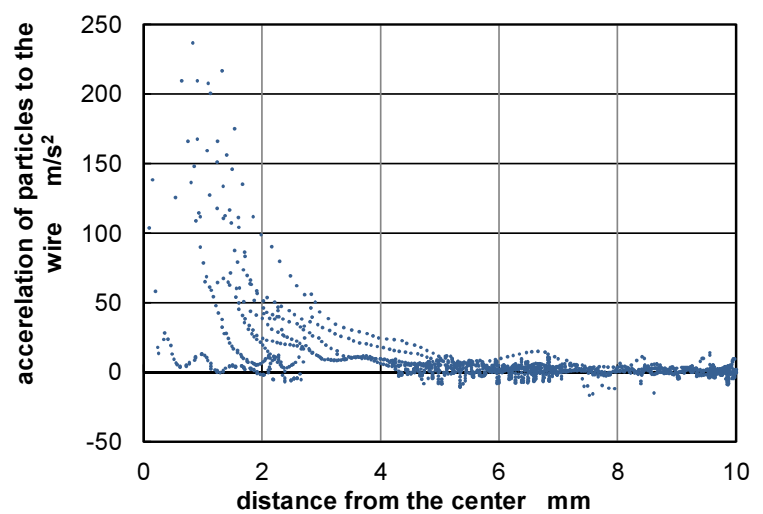

(a) in atmosphere pressure $\left(10^{5} \mathrm{~Pa}\right)$ air, $4.5 \mathrm{kV}$

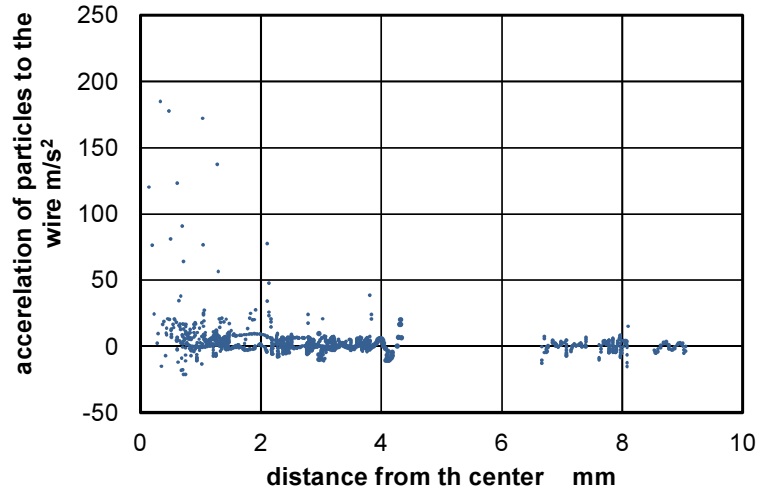

(b) in 700 Pa air, $0.6 \mathrm{kV}$

Figure 7. Measured acceleration to the wire of randomly selected particles in the wire-to-plate system.

\section{B. Numerical Calculation of Particle Motion}

Particle dynamics was simulated to investigate the difference between the particle motion at $10^{5} \mathrm{~Pa}$ and that at $700 \mathrm{~Pa}$. Particle motion was calculated based on a simple motion equation.

$$
\begin{aligned}
& m \ddot{x}=q E_{x}+2 \pi \varepsilon_{0} \varepsilon_{r} \frac{1-\varepsilon_{r}}{1+2 \varepsilon_{r}}\left(\frac{d}{2}\right) \operatorname{grad} E_{x}{ }^{2}-\frac{3 \pi \eta d}{C_{c}} \dot{x} \\
& m \ddot{y}=m g-\frac{3 \pi \eta d}{C_{c}} \dot{y}
\end{aligned}
$$

where $x$ is the lateral coordinate from the center of the wire electrode, $m$ is mass of the particle, $q$ is the charge of particle, $E_{x}$ is the electrostatic field in the lateral direction, $\varepsilon_{r}$ is the relative permittivity of the particle, $d$ is the diameter of the particle, $\eta$ is the viscosity of air $\left(1.82 \times 10^{-5} \mathrm{~Pa} \cdot \mathrm{s}\right), C_{c}$ is the Cunningham correction factor, ${ }^{18} y$ is the axial coordinate from the top of the system, and $g$ is the gravitational acceleration. The electrostatic field was calculated by the finite difference method, by reproducing the geometry and boundary conditions of the actual system. The first, second, and third terms in the right-hand side of Equation (3) are the Coulomb force, dielectrophoresis force, ${ }^{19}$ and air drag, respectively. The first and second terms in the right-hand side of Equation (4) are the gravitational force and the air drag, respectively. Equations (3) and (4) are numerically calculated using the Runge-Kutta method.

The charge of the particle is one of the critical parameters in the numerical calculation. Although substantial efforts have been devoted on the charging of particulates, it is still unclear. It depends on many parameters, such as particle size, composition, atmosphere, and collision. The charge is not identical but distributes in wide range, from positive to negative. ${ }^{7}$ Therefore, we adopted the typical values measured by the free-fall method. ${ }^{7,20}$ In this method, the particles were supplied at the center of the parallel plate electrodes, to which high DC voltage was applied. The 
particles fell down, moving right or left from the center depending on the polarity and charge of the particle. A picture of all fallen particles that settled on an adhesive sheet on the grounded lower plate was taken using a highprecision scanner (CanoScan 9000F, Canon), and the charge of the particle was deduced implicitly using their distance from the centerline. The measured result at $10^{5} \mathrm{~Pa}$ indicated that although the charge densities were distributed in a wide range both positively and negatively, the averaged values of positively and negatively charged small particles were approximately $+0.03 \mu \mathrm{C} / \mathrm{g}$ and $-0.13 \mu \mathrm{C} / \mathrm{g}$. On the other hand, at $700 \mathrm{~Pa}$, the respective values were +0.02 $\mu \mathrm{C} / \mathrm{g}$ and $-0.02 \mu \mathrm{C} / \mathrm{g}$. Therefore, the charge density of the particle at $10^{5} \mathrm{~Pa}$ was assumed to be $-0.1 \mu \mathrm{C} / \mathrm{g}$, and at the other two cases, $+0.001 \mu \mathrm{C} / \mathrm{g}$ and $-0.01 \mu \mathrm{C} / \mathrm{g}$, as calculated at $700 \mathrm{~Pa}$.

Figure 8 shows the calculated trajectories of the particles. It was predicted that particles fed at $9.5 \mathrm{~mm}$ from the wire were attracted to the wire electrode at $10^{5} \mathrm{~Pa}$. On the other hand, the effective region at $700 \mathrm{~Pa}$ was $1.7 \mathrm{~mm}$ from the wire for the negatively charged particle and only $0.5 \mathrm{~mm}$ for the positively charged particle, values which were much smaller than that at $10^{5} \mathrm{~Pa}$. These calculated results coincided with the experimental results described above. Considering all the experimental and numerical results, it was assumed that the low charge density of particles resulted in the low Coulomb force, and because the dielectrophoresis force was dominant, the particles were deposited on the wire at $700 \mathrm{~Pa}$. It was assumed that the low charge density was caused by the discharge between particles during the evacuation process, because the threshold voltage of the spark discharge was low at low pressure, based on Paschen's law. ${ }^{17}$

Calculated particle motions in the Martian gravity are added in Fig. 8 (b) and (c), designated in broken blue curves. Particles are attracted on the upper side of the wire electrode owing to the relatively low gravity on Mars, but fundamental characteristics in the Mars gravity is similar to that in the Earth gravity.

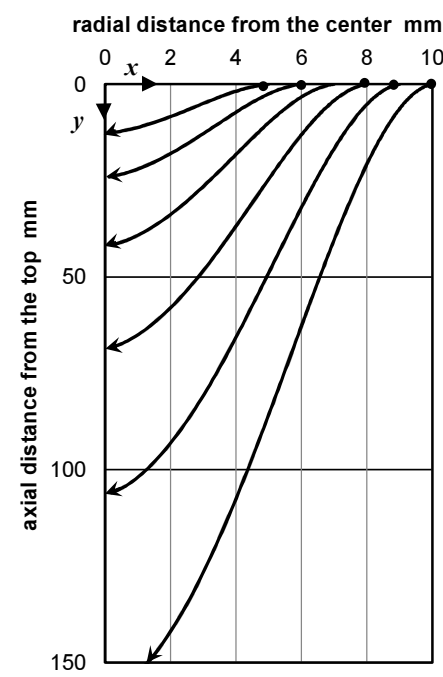

(a) in atmosphere pressure $\left(10^{5} \mathrm{~Pa}\right)$ air $4.5 \mathrm{kV},-0.1 \mu \mathrm{C} / \mathrm{g}$

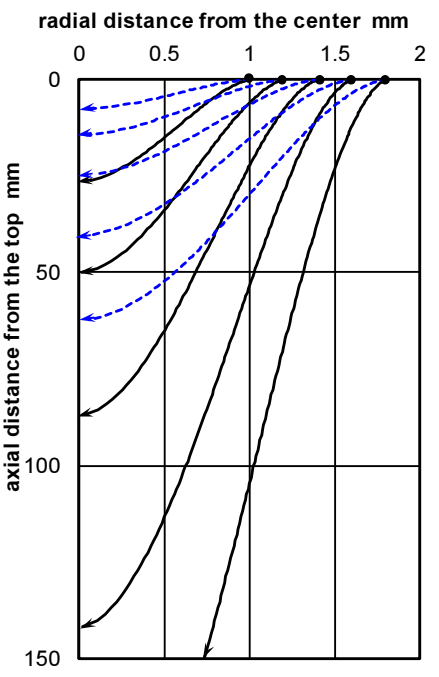

(b) in 700 Pa air, $0.6 \mathrm{kV}$ $0.6 \mathrm{kV},-0.01 \mu \mathrm{C} / \mathrm{g}$

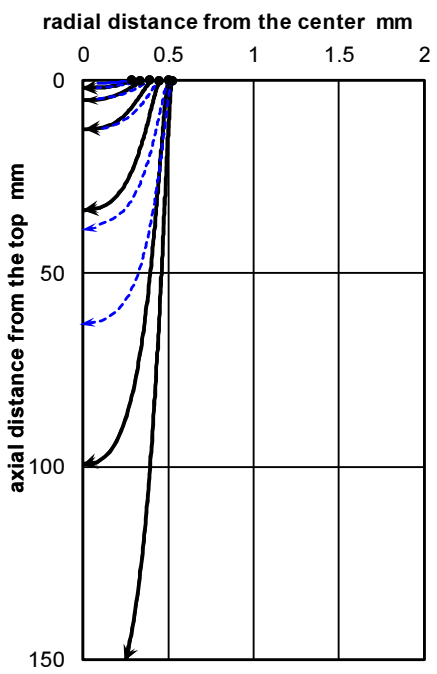

(c) in $700 \mathrm{~Pa}$ air $0.6 \mathrm{kV},+0.001 \mu \mathrm{C} / \mathrm{g}$

Figure 8. Calculated trajectories of particles. Solid black curves are trajectories in the Earth gravity and broken blue curves are those in the Mars gravity. (parameter: initial position of particle, $d=20 \mu \mathrm{m}$ )

\section{Cleaning of Trapped Dust}

Figure 9 shows time dependent characteristics of dust collection performance in $700 \mathrm{~Pa}$ air. Figure 9 (a) is weight of slipped dust from the precipitator versus the operation time. The feed rate was deduced from the derivative of the weight of slipped dust without application of voltage, designated in red. The dust collection rate shown in Figure 9 (b) was determined by the rate of the slipped dust with the application of voltage divided by the feed rate. It is clearly seen that the performance was degraded very rapidly, because dust is collected on the fine wire electrode in the low-pressure air (700 Pa). Actually, cleaning of collected dust is one of the most serious issues in industrial electrostatic precipitators. However, fortunately, it is very easy to vibrate the wire electrode and to remove dust on the wire. We have successfully demonstrated the cleaning of dust by vibrating the wire. It was realized by a small unbalance motor attached at the top end of the wire. 


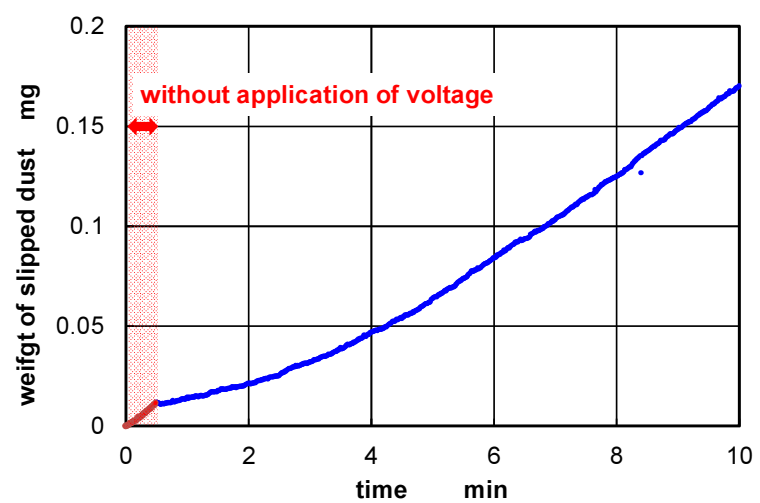

(a) weight of slipped dust versus operation time

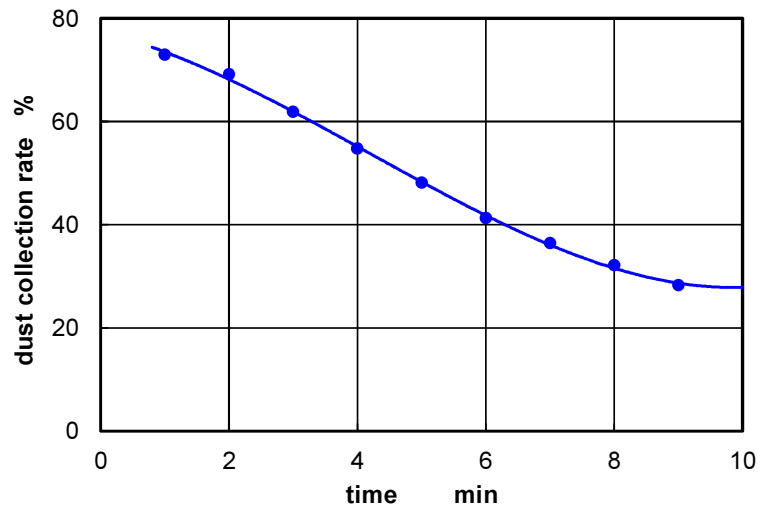

(b) dust collection rate versus operation time

Figure 9. Time dependent characteristics of dust collection performance in the parallel wire-to-plate system. (0.6 kV, in $700 \mathrm{~Pa}$ air)

\section{Conclusion}

An electrostatic precipitator was developed to remove dust from the $\mathrm{CO}_{2}$ gas to be used in ISRU for the production of oxygen in the Martian environment. The electrostatic precipitator is expected to make the atmospheric gas clean and usable for the oxygen extraction system. The technology has several advantages, such as low-pressure drop, low power consumption, and high collection efficiency. The setups that consist of the wire-to-cylinder electrode and wire-to-plate electrode were constructed to investigate the fundamental characteristics of the system. A preliminary experiment was conducted and it was found that performance at low pressure $(700 \mathrm{~Pa})$, which was selected to simulate the Martian environment was different from that at atmosphere pressure $\left(10^{5} \mathrm{~Pa}\right)$, i.e., (1) the applied voltage must be low because the values of corona onset and spark discharge voltage are low compared to those at $10^{5} \mathrm{~Pa},(2)$ the dust deposited on the wire electrode at $700 \mathrm{~Pa}$ while it deposited on the cylinder or plate electrodes in atmosphere pressure, (3) at corona discharge region no dust was collected in $700 \mathrm{~Pa}$ while the dust collection rate was almost $100 \%$ at $10^{5} \mathrm{~Pa}$. The mechanism of these phenomena was investigated by direct observation and numerical calculation of the particle motion, and it was clarified that lower applied voltages and lower charge density of the particles caused these characteristics. Although the performance was degraded very rapidly in low pressure atmosphere due to the deposition of dust on the fine wire electrode, it was demonstrated that it was very easy to vibrate the wire electrode and to remove dust on the wire. Countermeasures to realize high deposition performance of the system in the Martian atmosphere are under investigation.

\section{Acknowledgment}

The authors would like to express their gratitude to Ryotaro Sawai, Cheng Kedong, and Yuki Matsumoto (Waseda University) for their support in conducting the experiment.

\section{References}

${ }^{1}$ Sanders, G. B. and William E. L., "Integration of In-Situ Resource Utilization into lunar/Mars exploration through field analogs," Advances in Space Research, Vol. 47, No. 1, 2011, pp.20-29.

${ }^{2}$ Mantovani, J. G. and Townsend, I. I., "Planetary regolith delivery systems for ISRU," J. Aerospace Engineering, Vol. 26, No. 1, 2012, pp.169-175.

${ }^{3}$ Sanders, G. B., Araghi, K., Ess, K. M., Valencia, L. M., Muscatello, A. C., Calle, C. I., Clark, L. and Iacomini C., "Mars Atmosphere Resource Verification Insitu (MARVIN) - In Situ Resource Demonstration for the Mars 2020 Mission," AIAA SPACE 2014 Conference and Exposition, AIAA 2014-4235, Washington, DC, 2014.

${ }^{4}$ Kawamoto, H. and Adachi, M., "Electrostatic Particle-Size Classification of Lunar Regolith for In-Situ Resource Utilization," AIAA SciTech 2014, Washington, DC, 2014.

${ }^{5}$ Adachi, M., Nogami, K. and Kawamoto, H., "Transport of Regolith Utilizing Dielectric Elastomer Actuator for In-Situ Resource Utilization on Moon and Mars," 30th ISTS: International Symposium on Space Technology and Science, 2015-k-26, 2015

${ }^{6}$ Kawamoto, H., Shigeta, A. and Adachi, M., "Utilizing Electrostatic Force and Mechanical Vibration to Obtain Regolith Sample from the Moon and Mars,” J. Aerospace Engineering, Vol. 29, Issue 1, 2016, 04015031-1-6.

${ }^{7}$ Adachi, M., Moroka, H., Kawamoto, H., Wakabayashi, S. and Hoshino, T., "Particle-Size Sorting System of Lunar Regolith Using Electrostatic Traveling Wave,” Proceedings of the 2016 Electrostatics Joint Conference, B5, 2016. 
${ }^{8}$ Greeley, R., et al., "Active dust devils in Gusev crater, Mars: observations from the Mars exploration rover spirit." J. Geophysical Research, Vol. 111, E12S09, 2006.

${ }^{9}$ Calle, C. I., "The electrostatic environments of Mars and the Moon", J. Physics: Conference Series, 301, 2011, 012006.

${ }^{10}$ Pang, H. L., Atten, P. and Reboud, J., "Corona Discharge and Electrostatic Precipitation in Carbon Dioxide Under Reduced Pressure Simulating Mars Atmosphere", IEEE Trans. Industry Applications, Vol. 45, No. 1, 2009, pp.50-58.

${ }^{11}$ Calle, C. I., Thompson, S. M., Cox, N. D., Johansen, M. R., Williams, B. S., Hogue, M. D. and Clements, J. S., "Electrostatic precipitation of dust in the Martian atmosphere: Implications for the utilization of resources during future manned exploration missions", J. Physics: Conference Series, 327, 2011, 012048.

${ }^{12}$ Clements, J. S., et al., "Development of an electrostatic precipitator to remove Martian atmospheric dust from ISRU gas intakes during planetary exploration missions," IEEE Transactions on Industry Applications, Vol. 49, No. 6, 2013, pp.2388-2396.

${ }^{13}$ Calle, C. I., et al., "An electrostatic precipitator system for the Martian environment,” J. Electrostatics, Vol. 71, No. 3, 2013, pp.254-256.

${ }^{14} \mathrm{http} / / /$ www.appie.or.jp/en/testpowders/jis-test-powders1jis-z-8901-jis-7811-kanto-japanese-loam/ [cited 21 February 2017].

${ }^{15}$ Rieder, R., et al., "The chemical composition of Martian soil and rocks returned by the mobile alpha proton X-ray spectrometer: Preliminary results from the X-ray mode," Science, Vol. 278, 1997, pp.1771-1774.

${ }^{16}$ Allen, C. C., Jager, K. M., Morris, R. V., Lindstrom, D. J., Lindstrom, M. M. and Lockwood, J. P., "Martian soil simulant available for scientific, educational study," Eos, Vol. 79, No. 34, 1998, pp.405-409.

${ }^{17}$ Williams, E. M., The Physics and Technology of Xerographic Processes, Krieger, Huntington, NY, 1993.

${ }^{18}$ Munakata, T., "Note on Cunningham correction factor." Journal of the Society of Powder Technology, Vol. 40, 1990, pp.91-97 (in Japanese).

${ }^{19}$ Jones, T. B., Electromechanics of particles, Cambridge University Press, 1975, pp.5-8.

${ }^{20}$ Kawamoto, H., Seki, K. and Kuromiya, N., "Mechanism on Traveling-Wave Transport of Particles," J. Phys. D: Appl. Phys., Vol. 39, 2006, pp.1249-1256. 